\section{REVISTA}

Revista Educación

ISSN: 0379-7082

ISSN: 2215-2644

revedu@gmail.com

Universidad de Costa Rica

Costa Rica

\title{
Análisis de la matricialidad de los programas educativos de pregrado de la Red Universitaria de Jalisco
}

Guerrero-Herrera, Juan Fernando; Corona-Dueñas, José Asunción

Análisis de la matricialidad de los programas educativos de pregrado de la Red Universitaria de Jalisco

Revista Educación, vol. 43, núm. 1, 2019

Universidad de Costa Rica, Costa Rica

Disponible en: http://www.redalyc.org/articulo.oa?id=44057415022

DOI: https://doi.org/10.15517/revedu.v43i1.28868

Esta obra está bajo una Licencia Creative Commons Atribución-NoComercial-SinDerivar 3.0 Internacional. 


\section{Análisis de la matricialidad de los programas educativos de pregrado de la Red Universitaria de Jalisco}

Analysis of the matriciality of undergraduate educational programs of the University Network of Jalisco

Juan Fernando Guerrero-Herrera

DOI: https://doi.org/10.15517/revedu.v43i1.28868

Universidad de Guadalajara, México

Redalyc: http://www.redalyc.org/articulo.oa?id=44057415022

fegueh@gmail.com

iD http://orcid.org/0000-0001-5812-8179

José Asunción Corona-Dueñas

Universidad de Guadalajara, México

jcoronadueas@gmail.com

iD http://orcid.org/0000-0001-8603-7856

Recepción: 09 Mayo 2018

Aprobación: 14 Diciembre 2018

\section{RESUMEN:}

Este artículo presenta una evaluación diagnóstica del modelo académico de carácter matricial implementado en la Universidad de Guadalajara. Muestra los resultados de un benchmarking interno sustentado en la aplicación de una encuesta censal a los coordinadores de carrera (de programas de pregrado) que integran la Red Universitaria de Jalisco. El trabajo tiene como finalidad revisar el nivel de matricialidad académica de los programas de pregrado, identificar áreas de oportunidad que permitan fortalecer la matricialidad académica y aportar elementos que permitan elevar la calidad de dichos programas. El benchmarking interno permite realizar un análisis y evaluación diagnóstica que puede servir de referente para tratar la matricialidad académica de los programas de pregrado de las denominadas macrouniversidades. Para obtener la información de análisis se aplicó una encuesta estandarizada con 53 reactivos a nivel censal. La información se recabó a través de una plataforma virtual a los 200 coordinadores de carrera de los 15 Centros Universitarios (seis metropolitanos y nueve regionales) y Sistema de Universidad Virtual (SUV) que conforman la Red Universitaria de Jalisco. El resultado muestra que en la Universidad de Guadalajara (en su Red Universitaria) el nivel de matricialidad de los programas de pregrado se encuentra en proceso de desarrollo y consolidación, en virtud de que solo el $48.3 \%$ del total de departamentos académicos participa en la oferta de las unidades de aprendizaje que integran los planes de estudios. Asimismo, los niveles de matricialidad de los programas de pregrado por tipo de centro universitario muestran diferencias significativas, pues en los centros universitarios temáticos el promedio de participación es del 70\%, mientras que en los mutidisciplinares es del 39\%.

Palabras Clave: Pregrado, Matricialidad, Interdisciplina, Multidisciplina, Transdisciplina.

\section{Abstract:}

This article presents a diagnostic evaluation of the academic model of matrix character implemented at the University of Guadalajara. It shows the results of an internal benchmarking based on the application of a census survey to the career coordinators (of undergraduate programs) that integrate the University Network of Jalisco. The aim of the work is to check the level of academic matriciality of the undergraduate programs, identify areas of opportunity that allow for the strengthening of academic matriciality and provide elements that allow the quality of such programs. The internal benchmarking allows to perform a diagnostic analysis and evaluation that can serve as a reference for try the academic matriciality of the undergraduate programs of the so-called macro universities. To obtain the analysis information, a standardized survey was applied with 53 reagents at census level. The information was collected through a virtual platform to the 200 career coordinators of the 15 University Centers (6 metropolitan and 9 regional) and Virtual University System (SUV) that make up the University Network of Jalisco. The result shows that in the University of Guadalajara (in his University Network) the level of matriciality of the undergraduate programs is in the process of development and consolidation, since only $48.3 \%$ of the total of academic departments participate in the offer of the units of learning that integrate the curricula. Likewise, the levels of matriciality of the undergraduate programs by type of university center show significant differences, since in thematic university centers the participation rate is $70 \%$, while in the multidisciplinary programs it is $39 \%$.

KEYWORDs: Undergraduate, Matriciality, Interdiscipline, Multidiscipline, Transdiscipline. 


\section{INTRODUCCIÓN}

La forma en que las universidades se han organizado para ofrecer docencia y aprendizaje ha evolucionado con la finalidad de cumplir de forma eficaz y pertinente con su función social. En la actualidad la mayoría de las Instituciones de Educación Superior están cambiando la forma de trasmitir, compartir y generar conocimiento con la intención de responder de mejor manera al entorno y coadyuvar a la solución de los problemas que le aquejan.

En la actualidad diversas universidades han adoptado la organización académica de tipo matricial con el propósito de atender el reto que representa la educación superior. Este es el caso de la Universidad de Guadalajara, institución que en la década de los noventa implementó el sistema universitario en red y el modelo departamental de carácter matricial y flexible para el desarrollo de sus funciones educativas y académicas.

En la educación superior la implementación del modelo matricial es de fundamental importancia en virtud de que permite el tránsito de la educación de tipo disciplinar a la de carácter inter, multi o transdisciplinar. Sin embargo, la adopción de este modelo conlleva muchas implicaciones, el proceso de implementación impacta el funcionamiento de la universidad en muchos sentidos, por ello y con la finalidad de apoyar la mejor toma de decisiones académicas se hace necesario evaluar periódicamente el funcionamiento del modelo matricial en todas sus aristas y vertientes.

Con la finalidad de contribuir a dicho cometido, se realiza el análisis de la matricialidad de los programas de pregrado que integran la Red de la Universidad de Guadalajara.

\section{Problematización}

A partir de la década de 1930 en el ámbito de la Educación Superior inicio el empleo frecuente de los términos multidisciplina, interdisciplina y transdisciplina a nivel nacional e internacional, sin embargo el tipo de estructura y organización académica implementada en las Universidades e Instituciones de Educación Superior para instrumentar con éxito estos enfoques educativos, así como la evaluación de su funcionamiento operativo han sido poco tratados, en virtud de ello el presente tiene como intención lograr un acercamiento que permita valorar la eficiencia funcional del modelo académico de tipo matricial y su articulación con los enfoques referidos en la Universidad de Guadalajara (en su Red Universitaria).

\section{OBjetivos}

La Universidad de Guadalajara se ubica en las denominadas macrouniversidades por el número de estudiantes que tiene matriculados ${ }^{[1]}$ y el número de programas de pregrado que oferta. A partir de la creación de la Red Universitaria la oferta de programas de pregrado y el número de coordinadores de carrera ha crecido considerablemente, llegando a 202 y 183 , respectivamente.

A poco más de 20 años de implementado el modelo matricial no se cuenta con un estudio que permita conocer su grado de consolidación. Con la intención de abonar a este cometido se realizó una evaluación diagnóstica que tiene las finalidades siguientes:

a) Conocer los avances en el nivel de matricialidad ${ }^{[2]}$ de los programas de pregrado de la Red Universitaria.

b) Ubicar la forma en que se vinculan los programas de pregrado y coordinaciones de carrera con los Departamentos académicos.

c) Identificar elementos restrictores de la matricialidad académica de los programas de pregrado. 
d) Sugerir áreas de oportunidad para el fortalecimiento de la matricialidad académica de los programas de pregrado.

e) Recomendar acciones para fortalecer la implementación de los enfoques académicos de tipo multidisciplinar, interdisciplinar y/o transdisciplinar.

Los objetivos se plantean atendiendo el supuesto de que la organización académica de carácter matricial permite la adquisición, generación, aplicación y desarrollo de conocimientos de forma conjunta entre disciplinas de estudio, y por consiguiente, facilita el tránsito, implementación y funcionamiento de esquemas educativos y académicos de carácter multidisciplinar, interdisciplinar y/o transdisciplinar.

\section{REFERENTE TEÓRICO}

En la literatura educativa encontramos diversas referencias que plantean la importancia de incorporar la inter, multi o transdisciplina al ámbito de la educación superior, así como los retos que se deben atender; principalmente los relacionados con nuevas demandas, necesidades y requerimientos de aprendizaje, enseñanza e investigación; y su previsible impacto tanto en la organización educativa de las Universidades e Instituciones de Educación Superior, como en la implementación de estrategias y acciones para su actualización y/o reorganización.

A continuación se citan, a manera de ejemplo, algunos referentes teóricos que tratan la temática.

Del Rio en el año 2002 plantea que la generación y la aplicación de conocimiento se caracterizan por cuatro grandes tendencias, y una de ellas es "La creciente importancia de los métodos y procedimientos de atacar problemas en los que convergen varias disciplinas; en otras palabras, la cada vez mayor relevancia de la interdisciplina y la multidisciplina" (p.2). Adicional a lo anterior menciona que "La creciente importancia de las interdisciplinas en la educación superior y en la generación de nuevo conocimiento obliga a que los planes de estudio y de investigación contengan cada día más elementos de una mayor diversidad de disciplinas" (p. 2).

Luengo en el año 2003 señala que la UNESCO identifica tres principales desafíos en los sistemas de educación superior, y argumenta que

Estos desafíos implican repensar el papel y misión de la educación superior así como intentar superar sus retos desde nuevos enfoques y establecer propuestas alternativas para su avance futuro, lo que reclama la participación activa de los diversos actores involucrados. Es aquí, donde creemos que la perspectiva de la interdisciplina y la transdisciplina, acompañadas por el pensamiento complejo, pueden ofrecer una visión o alternativa para enfrentar los retos actuales de la educación superior... (pp. 24-25).

La UNESCO en el año 2009 en la declaración de resultados de la Conferencia Mundial de la Educación Superior, en el apartado correspondientes a la Responsabilidad Social de la Educación Superior plantea que "Los centros de educación superior, en el desempeño de sus funciones primordiales (investigación, enseñanza y servicio a la comunidad) en un contexto de autonomía institucional y libertad académica, deberían centrarse aún más en los aspectos interdisciplinarios...” (p. 2).

Escobar en el año 2010, en relación con los desafíos que expone la Educación Superior y la Investigación argumenta que

Los problemas fundamentales que enfrenta la humanidad, obligan a estudiarlos como un todo, demandando el concurso de todas las potencialidades del conocimiento humano, y exigiendo enfocarlos como complejos, inseparables y retroalimentados; de tal forma que surge la necesidad de abordar una visión integral e interdisciplinaria para resolverlos, que plantea cambios en la educación y la investigación con nuevos enfoques ínter y transdisciplinarios (p.165).

Luengo en el año 2012, con respecto a la problemática universitaria menciona que 
La inexistente o lenta reacción de las universidades para organizarse y poder generar conocimientos inter y transdisciplinares que den respuestas a las problemáticas múltiples de la sociedad, está favoreciendo a que nuestras instituciones dejen de ser consideradas como las únicas, instancias de producción de conocimientos (p.23).

Y haciendo referencia a Thompson et ál. (2001), señala

...todavía la transdisciplina (y aún la interdisciplina) no es vista como una forma alternativa y valiosa de organizar el trabajo universitario. Además, no se considera su potencial como un criterio para evaluar a las instituciones de investigación del sistema educativo y científico de nuestro país (p. 23).

Ardines (s.f.), con respecto a la enseñanza e investigación en la educación superior refiere que

El cambio creativo de la enseñanza universitaria y la investigación exige cada vez con mayor fuerza un acercamiento a la enseñanza interdisciplinaria. Esto no plantea la necesaria destrucción de la enseñanza organizada por disciplinas, sino de enseñar estas en función de las relaciones dinámicas con otras disciplinas y con los problemas de la sociedad (párr.4).

La ANUIES en el año 2018 en su propuesta para renovar la educación Superior en México, de manera particular en lo correspondiente a la expansión e innovación de la Educación Superior, precisa que

Los modelos educativos en el mundo están cambiando de manera acelerada. Se transita de la premisa de estabilidad a la turbulencia en el entorno; de la idea de permanencia de los saberes a la aceptación de su rápida obsolescencia; del abordaje rígido y disciplinario al flexible e interdisciplinario;...” (p. 31).

Asimismo, con respecto al desafío que enseña el incremento de la competitividad nacional en el marco de la Cuarta Revolución Industrial, la ANUIES argumenta que las y los estudiantes de las Instituciones de Educación Superior

Deben egresar con bases sólidas y dispuestos a mantenerse aprendiendo a lo largo de su vida profesional para estar en condiciones de desempeñarse con solvencia en trabajos que aún no existen, usar tecnologías que no han sido inventadas, resolver problemas inéditos y aprender a trabajar colaborativamente en ambientes multiculturales y multidisciplinarios (ANUIES, 2018, p.42).

\section{CONCEPTUALIZACIÓN}

Como nivel educativo, el pregrado tiene diferentes acepciones según el país de que se trate. En Estados Unidos se otorga el grado a partir de maestrías y doctorados que brindan una formación especializante, de ahí que a la formación previa orientada fundamentalmente a la adquisición de conocimientos generales de las disciplinas se le denomine pregrado. A partir de los acuerdos de Bolonia en la Unión Europea, el pregrado también conocido como licenciatura o grado está conformado por programas de estudio profesionalizantes que preparan a las y los estudiantes para su incorporación al mercado laboral.

En México se utiliza comúnmente el término de licenciatura como sinónimo del pregrado, y se ubica en el nivel de educación superior. La Ley para la Coordinación de la Educación Superior, en el artículo 3 señala:

El tipo educativo superior es el que se imparte después del bachillerato o de su equivalente, que comprende la educación normal, tecnológica y universitaria que incluye carreras profesionales cortas y estudios encaminados a obtener los grados de licenciatura, maestría y doctorado, así como cursos de actualización y especialización (Congreso de los Estados Unidos Mexicanos, 1978, p. 1).

En la Universidad de Guadalajara el pregrado comprende la formación previa a la especialidad, maestría y doctorado, e incluye los niveles de licenciatura, técnico superior universitario (TSU) y nivelaciones a licenciatura. Se trata, en esencia, de una formación que habilita para el ejercicio de una profesión, en este sentido, demanda el dominio de habilidades y conocimientos generales sobre una disciplina, de sus interrelaciones con otras, que permita al egresado poseer un conjunto de competencias para su desempeño profesional. 


\section{Programas de pregrado por enfoque disciplinar}

Las formas de conocer nuestro entorno, atender nuestras necesidades y solucionar los problemas de nuestro medio han evolucionado a través del tiempo, dando surgimiento a nuevas disciplinas científicas, actualización o recomposición teórica y práctica de las existentes; abordaje de nuevas escuelas y paradigmas del pensamiento; y tránsito de enfoques disciplinarios (unidisciplinar, multidisciplinar, interdisciplinar y transdisciplinar), y por consiguiente, la aplicación de nuevas formas de organizar el conocimiento para el aprendizaje, la enseñanza y la investigación.

Por lo anterior, se hace necesario ubicar al programa educativo de pregrado de acuerdo al concepto de cada enfoque disciplinar. En la Tabla No.1 se describe la definición y caracterización por tipo de enfoque.

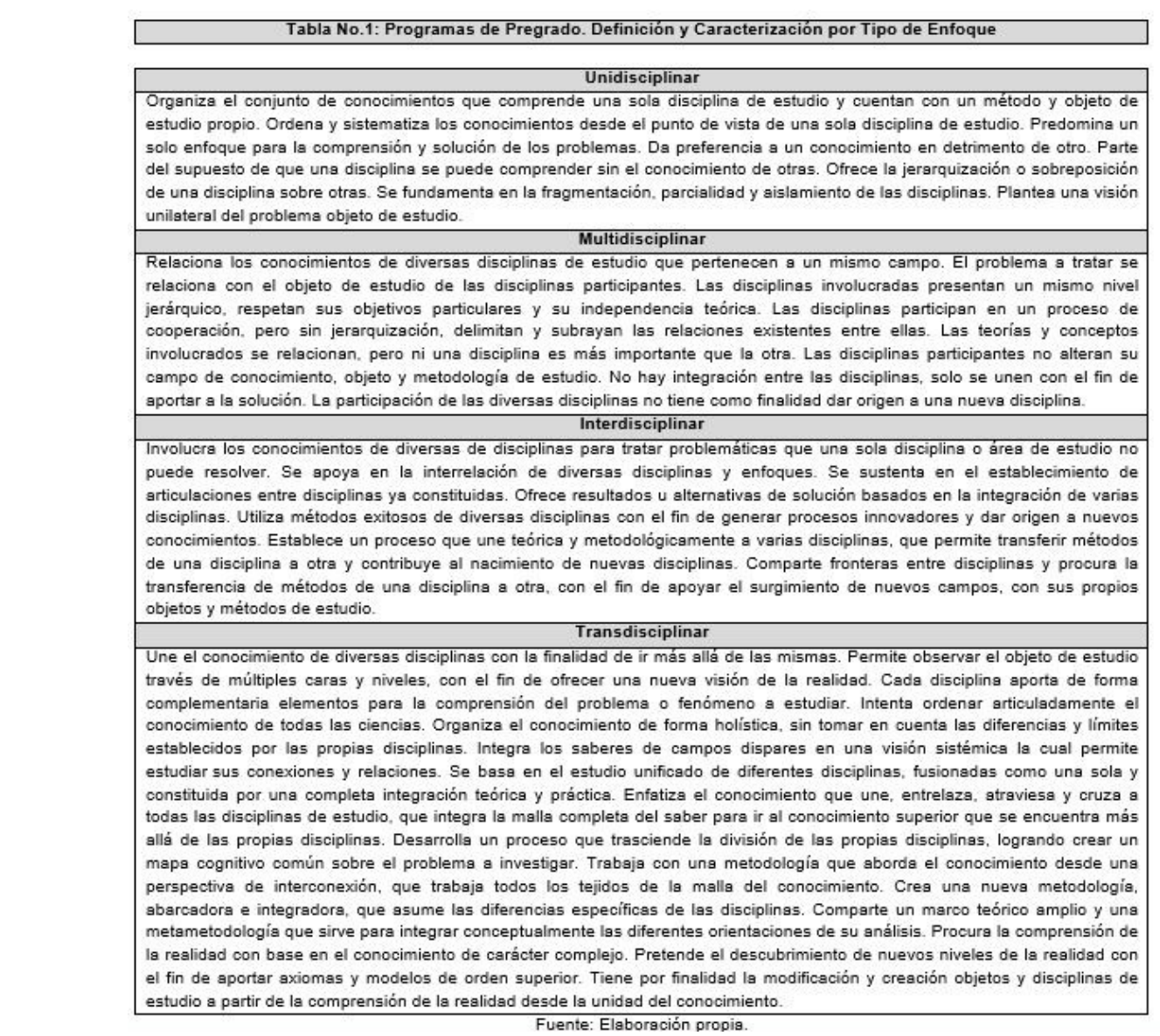

Tabla No.1: Programas de Pregrado. Definición y Caracterización por Tipo de Enfoque Fuente: Elaboración propia.

\section{MARCo CONTEXTUAL}

La Universidad de Guadalajara en la década de los noventa adoptó el modelo en red integrado por centros universitarios que funcionan como dependencias académicas, docentes y de investigación desconcentradas, con una estructura departamental organizada por campos del conocimiento y coordinaciones de carrera (de programas de pregrado) que se nutren académicamente de los distintos departamentos académicos.

Para atender el nivel de estudios superior la Red Universitaria de Jalisco a la fecha cuenta con 15 centros universitarios y el Sistema de Universidad Virtual (SUV), mismos que en conjunto comprenden 
15 secretarias académicas, una dirección académica, 41 Divisiones, 148 Departamentos académicos, 183 coordinadores de carrera, y ofrecen a la sociedad 202 programas de pregrado.

Del total de centros universitarios 6 son de carácter temático y se ubican en la Zona Metropolitana de Guadalajara, y 9 son de carácter multidisciplinario y se ubican en las regiones del interior del estado de Jalisco. [3]

En la Tabla No.2 se pueden observar los departamentos y programas educativos de pregrado que integran y ofrece cada centro universitario metropolitano y regional.

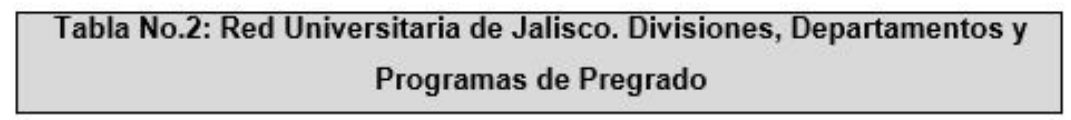

\begin{tabular}{|c|c|c|c|}
\hline Centro Universitario & Divisiones & Departamentos & $\begin{array}{c}\text { PE de } \\
\text { Pregrado }\end{array}$ \\
\hline CUAAD & 3 & 12 & 11 \\
\hline CUCBA & 3 & 10 & 5 \\
\hline CUCEA & 3 & 14 & 11 \\
\hline CUCEI & 3 & 12 & 15 \\
\hline CUCS & 3 & 19 & 13 \\
\hline CUCSH & 5 & 23 & 16 \\
\hline CUALTOS & 2 & 5 & 13 \\
\hline CUCINEGA & 3 & 9 & 16 \\
\hline CUCOSTA & 3 & 10 & 17 \\
\hline CULAGOS & 2 & 4 & 12 \\
\hline CUCSUR & 2 & 8 & 14 \\
\hline CUNORTE & 2 & 4 & 11 \\
\hline CUSUR & 2 & 5 & 14 \\
\hline CUVALLES & 3 & 6 & 14 \\
\hline CUTONALA & 2 & 7 & 13 \\
\hline SUV & 0 & 0 & 7 \\
\hline Total & 41 & 148 & 202 \\
\hline
\end{tabular}

Fuente: Elaboración propia, con base en información del Sistema Integral de Información

y Administración Universitaria (SIIAU), mayo 2016.

Tabla No.2: Red Universitaria de Jalisco. Divisiones, Departamentos y Programas de Pregrado

Fuente: Elaboración propia, con base en información del Sistema Integral

de Información y Administración Universitaria (SIIAU), mayo 2016.

De los 202 programas educativos de pregrado; 77 son de carácter exclusivo, es decir, cada programa lo ofrece un solo centro universitario; y 37 son compartidos, es decir, los ofrece más de un centro universitario, por ejemplo, la carrera de abogado en la modalidad escolarizada la ofrecen 10 centros universitarios de la Red. Asimismo, los 202 programas de pregrado se dividen en 187 de licenciatura, 9 de técnico superior universitario y 6 de nivelación.

Por otra parte, los 202 programas educativos de pregrado están a cargo de 183 coordinadores de carrera, 167 coordinan un solo programa de pregrado y 16 coordinan más de uno. 


\section{Modelo matricial}

En la Universidad de Guadalajara el diseño y operación del modelo matricial incorpora dos piedras angulares, por una parte están los departamentos académicos que tienen adscritos a las y los docentes, y por otra, están los programas de pregrado con un coordinador de carrera y al cual están inscritos las y los estudiantes. En este modelo, los departamentos académicos ofrecen el número de asignaturas que de acuerdo a su especialidad abonan al plan de estudios (estructura curricular) de cada programa de pregrado, y los coordinadores de carrera son los responsables de observar el cumplimiento de los respectivos planes de estudios.

Este modelo permite a los programas de pregrado servirse académicamente de algunos o todos los departamentos académicos que integran un centro universitario. De tal forma que no hay docentes que atiendan a un solo programa de pregrado, sino que pertenecen a un departamento académico, y las asignaturas que imparten pueden impactar a una o a varias carreras. Así, bajo el modelo departamental de carácter matricial los programas de pregrado cruzan trasversalmente la estructura académica, tomando los contenidos necesarios de cada departamento académico.

Los centros universitarios que integran la Red Universitaria aplican el modelo matricial que permite la aplicación del enfoque interdisciplinar en los centros temáticos o metropolitanos, y multidisciplinar en los centros regionales, a través de los cuales los departamentos académicos ofrecen el conjunto de asignaturas que en teoría permiten adquirir los conocimientos y habilidades necesarios para el desarrollo de cada profesión o programa de pregrado.

En el ámbito de la administración educativa y jerarquía organizacional cada departamento académico se encuentra adscrito a una División y las Coordinaciones de Carrera dependen de la Secretaría Académica de cada Centro Universitario.

La Tabla No.3 muestra el funcionamiento matricial del Centro Universitario de Ciencias Económico Administrativas (CUCEA) de la Universidad de Guadalajara, ilustra a manera de ejemplo los departamentos académicos que participan en la oferta de asignaturas de cuatro programas educativos de pregrado. 


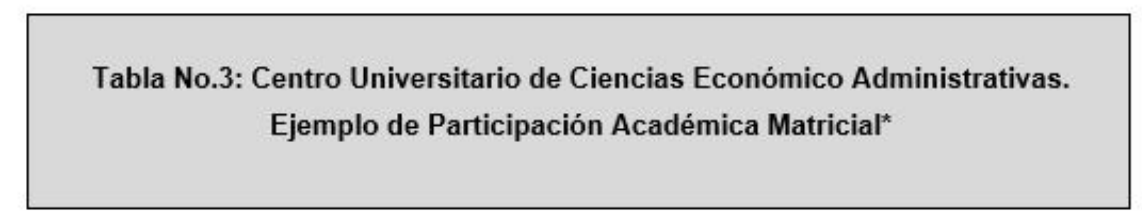

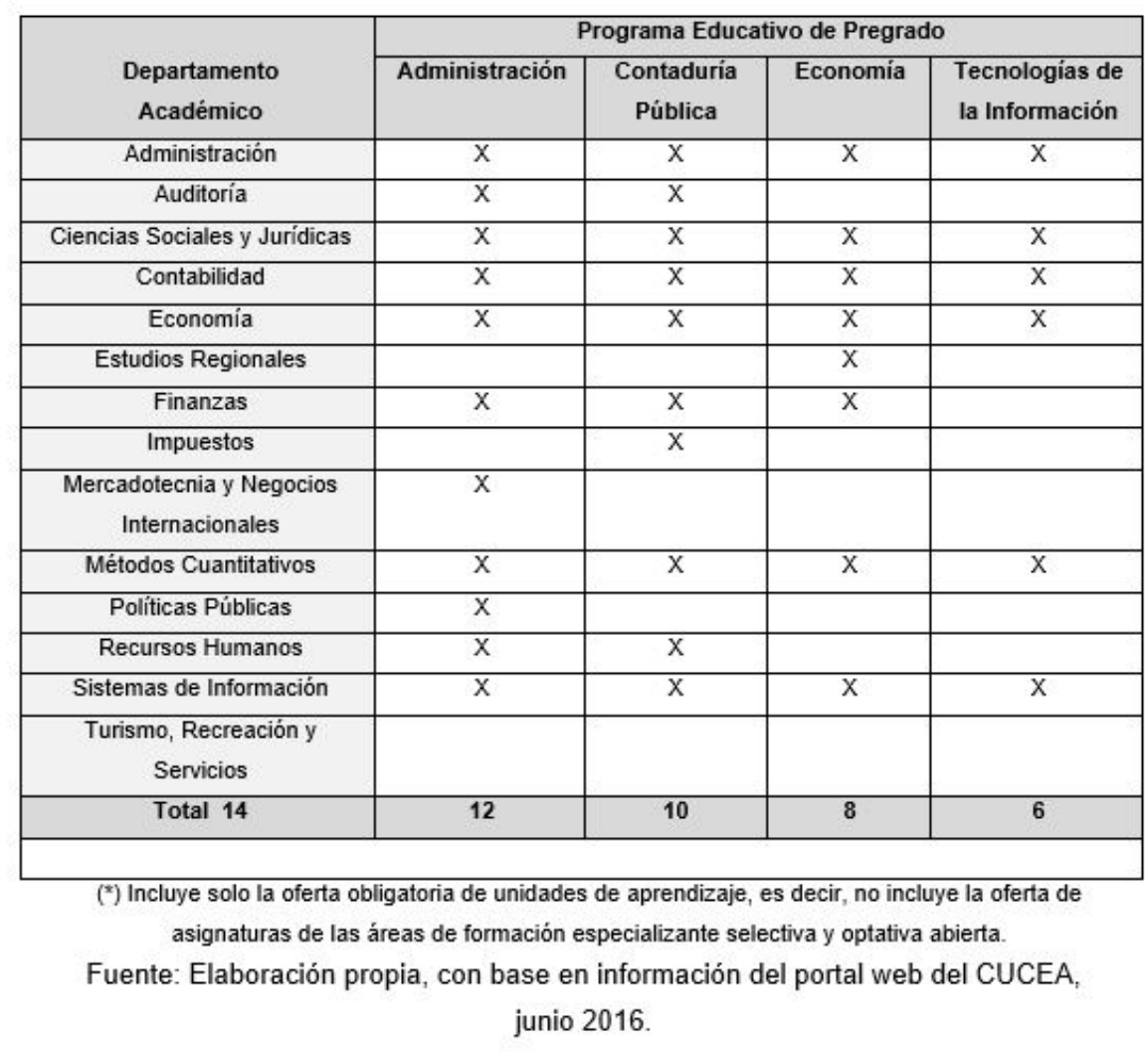

Tabla No.3: Centro Universitario de Ciencias Económico Administrativas Fuente: Elaboración propia, con base en información del portal web del CUCEA, junio 2016.

\section{Proceso metodológico}

El presente artículo aplica el análisis de benchmarking ${ }^{[4]}$ con el objetivo de conocer y contrastar la opinión de los coordinadores de carrera (de programa de pregrado), y con base en sus puntos de vista contar con una evaluación diagnóstica de la situación, con un análisis sustentado en el conocimiento, experiencia y prácticas de los coordinadores de carrera de la Universidad de Guadalajara ${ }^{[5]}$.

Para recabar la información y datos relativos a los objetivos planteados, los autores aplicaron una encuesta estandarizada con 53 reactivos a nivel censal (con preguntas cerradas y abiertas), y tomaron como sujetos de estudio al $100 \%$ de coordinadores de carrera de la Universidad de Guadalajara ${ }^{[6]}$.

El ejercicio involucró un universo de 200 coordinadores de carrera. La información se recabó a través de una plataforma virtual a los coordinadores de los 15 Centros Universitarios (seis metropolitanos y nueve regionales) y Sistema de Universidad Virtual (SUV) que conforman la Red Universitaria de Jalisco.

La validación de la coherencia interna y confiabilidad del instrumento se realizó aplicando una prueba piloto a 16 coordinadores (uno por cada centro universitario y SUV), con base en el programa Statistical Package for the Social Sciences (SPSS), dando como resultado un grado de correlación de las preguntas del 98\%. Las respuestas se operacionalizaron a través de escalas y se tabularon a través de frecuencias y promedios, 
para finalmente interpretar los resultados, explicar la información resultante y describir los resultados u hallazgos.

El tratamiento de la información comprende las siguientes categorías o niveles de análisis: Red Universitaria, centro universitario (metropolitano y regional), nivel educativo (licenciatura, nivelación y TSU), modalidad educativa (escolarizada, mixta y a distancia), y tipo de programa de pregrado (exclusivo o compartido) ${ }^{[7]}$.

El análisis se elaboró con base en la oferta de programas de pregrado del ciclo escolar $2015 \mathrm{~B}$, y comprende un universo de 200 programas de la Red Universitaria de Jalisco; 124 de centros universitarios regionales, 68 de centros universitarios metropolitanos y 8 del Sistema de Universidad Virtual.

\section{ANÁLISIS DE RESULTADOS}

Al solicitar a los coordinadores de carrera el número de departamentos académicos que integran su Centro Universitario (CU) de adscripción y el número de departamentos que ofrecen asignaturas al plan de estudios del programa de Pregrado que coordinan, el resultado promedio a nivel Red fue de 9.87 y 4.77 respectivamente. Asimismo, el promedio de los CU temáticos fue de 6.89 y el de los CU regionales de 3.85. El Gráfico 1 muestra el promedio de departamentos que brindan asignaturas a los programas de pregrado de cada CU, comparado contra el total de departamentos académicos del propio CU.

Al preguntar a los coordinadores de carrera si el funcionamiento de la coordinación a su cargo se apega al modelo departamental de carácter matricial, si el programa de pregrado que coordina se sirve de algunos o todos los departamentos académicos que integran el CU, se encontró que el $6.37 \%$ considera que nunca o casi nunca; el $18 \%$ que a veces y el $75.4 \%$ que siempre o casi siempre. El Gráfico 2 muestra el porcentaje de respuestas para cada CU.

Al cuestionar a los 46 coordinadores de carrera que consideraron, que la función del programa de pregrado no se apega al modelo departamental de carácter matricial. Se obtuvieron las respuestas siguientes: debido a que el programa es de apertura reciente (4.54\%); debido a la normatividad universitaria (18.1\%); debido a la falta de una apropiada incorporación (20.4\%); debido a que sigue operando el sistema rígido de escuelas y facultades (13.6\%); debido a que se trabaja con un solo departamento académico (34\%); por el tipo de administración ejercida (9.09\%). El Gráfico 3 muestra la distribución de respuestas para cada CU.

$\mathrm{Al}$ interrogar a los 46 coordinadores de carrera que manifestaron, que no había apego al modelo departamental de carácter matricial, su punto de vista sobre lo que podría faltar para incorporar plenamente el programa de pregrado que coordina a este modelo. Las respuestas fueron las siguientes: Gestión más efectiva por parte de autoridades y jefes de departamento académico (8.69\%); mayor apoyo de departamentos académicos y autoridades hacia las coordinaciones de carrera (19.5\%); mayor articulación funcional entre coordinación de carrera y departamentos académicos (32.6\%); que la oferta de unidades de aprendizaje sea más incluyente con los departamentos académicos del CU (34.7\%); otras acciones (4.34\%). El Gráfico 4 muestra el porcentaje de respuestas para cada CU.

Al cuestionar a los coordinadores de carrera, con cuál instancia se vinculan más estrechamente para el desarrollo de trabajos académicos correspondientes al programa de pregrado que coordinan, se obtuvieron las siguientes respuestas: Jefe de departamento académico 35.7\%; Director de división 12.5\%; Coordinación de posgrado $0.49 \%$; Coordinador general de pregrado $8.57 \%$; Secretaría académica $37.2 \%$ y otros 5.39\%.

Al preguntar a los coordinadores de carrera, si eran invitados a participar de forma regular en las sesiones de trabajo de los órganos colegiados de su CU, se encontró que solo el 38.5\% de ellos son invitados a participar de forma regular en sesiones de trabajo de los diferentes órganos colegiados.

Al solicitar a los 118 coordinadores de carrera, que manifestaron no ser invitados a participar en las sesiones de trabajo de los órganos colegiados, si consideraban que esta situación generaba vacíos para el óptimo desarrollo del programa de pregrado que coordinan. El $0.84 \%$ de ellos considera que esto nunca sucede, un 
$0.84 \%$ que casi nunca sucede; el $27.1 \%$ considera que esto sucede a veces; el $39.8 \%$ que esto sucede casi siempre y el $31.3 \%$ que esto sucede siempre.

$\mathrm{Al}$ interrogar a los coordinadores de carrera, si consideran importante que el coordinador forme parte de los órganos colegiados de su CU, se encontró que el 97\% lo consideran importante.

$\mathrm{Al}$ cuestionar a los coordinadores de carrera, en qué órganos colegiados de su CU deberían tener participación. Un 37.6\% considera que deberían participar en el colegio departamental de su departamento académico eje; $8.11 \%$ considera que en algunos colegios departamentales; $3.14 \%$ considera que en todos los colegios departamentales; $25.6 \%$ que en el consejo de división; $19.6 \%$ considera deberían participar en el consejo de centro y $5.75 \%$ que en otros.

$\mathrm{Al}$ consultar a los coordinadores de carrera, sobre la forma en que debería de ser su participación en los órganos colegiados, se encontró que el $86.7 \%$ considera que su participación debe de ser con voz y voto, y el 13.2\% considera que debería de ser solo con voz.

$\mathrm{Al}$ inquirir a los coordinadores de carrera, si el esquema de funcionamiento actual permitía procesar adecuadamente las directrices y lineamientos dirigidos a las coordinaciones de pregrado (solo 78 coordinadores de carrera respondieron esta pregunta), el resultado fue el siguiente: el $6.41 \%$ consideran que esto casi nunca ocurre; el 35.8\% que sucede a veces; el 37.1\% que casi siempre y el $20.5 \%$ que ocurre siempre.

Al cuestionar a los 62 coordinadores de carrera (que contestaron alguna de las tres primeras opciones de la pregunta anterior), sobre las razones por las cuales los lineamientos y directrices dirigidos a las coordinaciones no se procesan adecuadamente. El 9.67\% consideró que ello se debe a que la información es confusa; el 20.7\% a que la comunicación es lenta e imprecisa; el $18.4 \%$ a que la información se dispersa y desvirtúa; el 13.8\% a que la información tiende a perderse; el 19.8\% a que las instrucciones no se atienden con oportunidad; y el $17.5 \%$ manifestaron otros motivos.

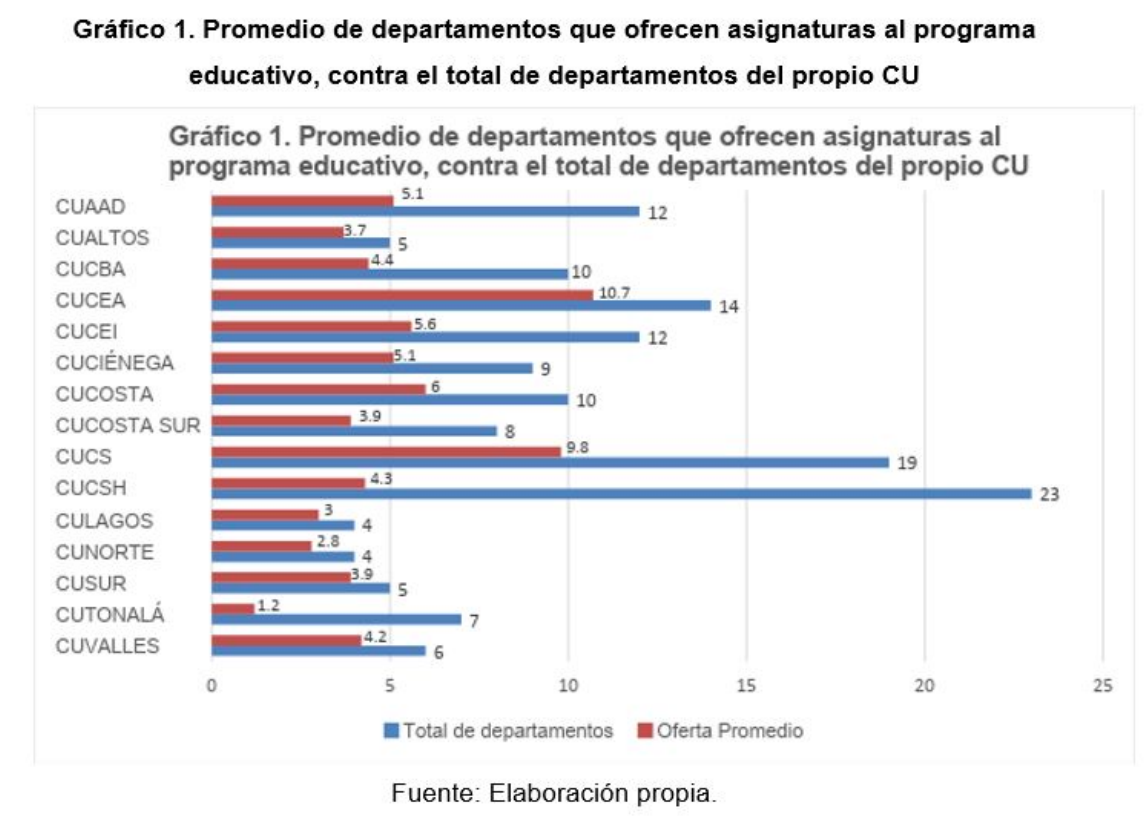

Gráfico 1. Promedio de departamentos que ofrecen asignaturas al programa educativo, contra el total de departamentos del propio $\mathrm{CU}$

Fuente: Elaboración propia 
Gráfico 2. Percepción de apego al modelo matricial de los PE de pregrado

Gráfico 2. Percepción de apego al modelo matricial de los PE de pregrado

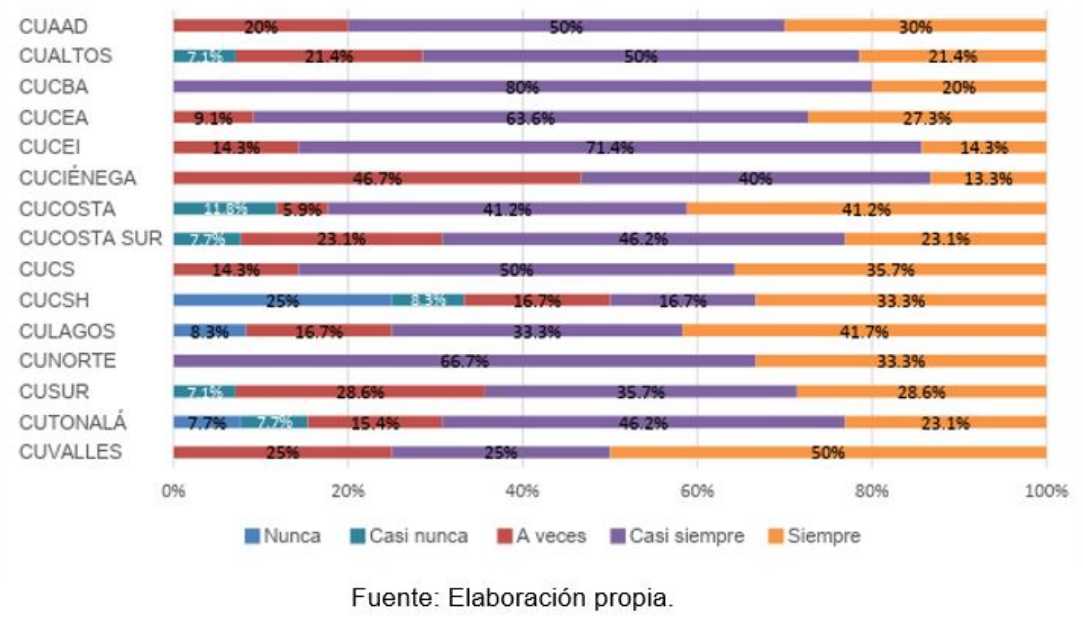

Gráfico 2. Percepción de apego al modelo matricial de los PE de pregrado Fuente: Elaboración propia

Gráfico 3. Motivos por los que los PE de pregrado no se apegan al modelo matricial

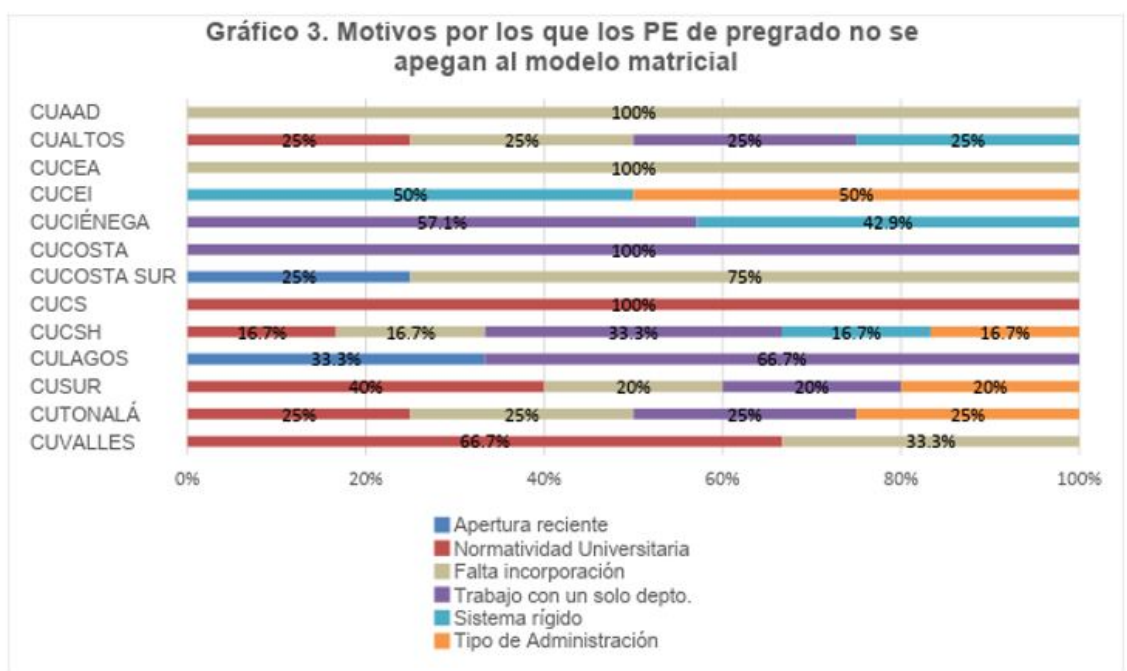

Fuente: Elaboración propia.

Gráfico 3. Motivos por los que los PE de pregrado no se apegan al modelo matricial Fuente: Elaboración propia 


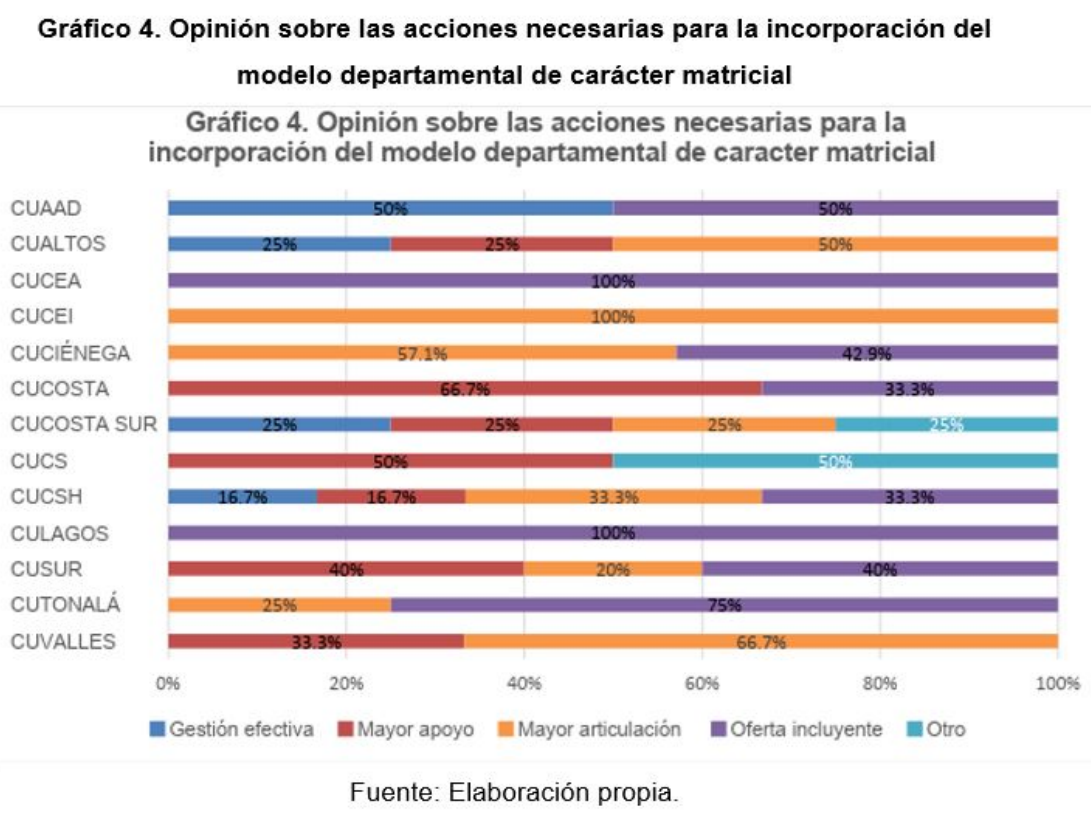

\section{Gráfico 4. Opinión sobre las acciones necesarias para la incorporación del modelo departamental de carácter matricial Fuente: Elaboración propia}

\section{CONCLUSIONES Y RECOMENDACIONES}

En la Universidad de Guadalajara (en su Red Universitaria) el modelo educativo sustentado en departamentos académicos está subutilizado y exhibe un nivel de desarrollo matricial asimétrico, ello se hace evidente desde el momento en que a nivel Red solo el $48.3 \%$ del total de departamentos académicos ofrece unidades de aprendizaje a los planes de estudio de los programas de pregrado, y al el hecho de que en los CU temáticos el promedio de participación de los departamentos académicos en los programas de pregrado es del $70 \%$, mientras que en los multidisciplinares es del $39 \%$.

A 20 años de constituida la Red Universitaria, el nivel de avance en el nivel de matricialidad de los programas de pregrado es medianamente aceptable, pues falta el $51.7 \%$ para llegar al nivel óptimo de matricialidad académica.

Para que los programas de pregrado tengan el máximo de matricialidad requieren integrar en su plan de estudios el conjunto de ciencias y disciplinas que abonan a la formación profesional de las y los estudiantes.

Cada programa de pregrado debe permitir la adquisición, aplicación y desarrollo de conocimientos relacionados con las disciplinas que integran los diversos campos y áreas de estudio y ofrecer aprendizajes articulados de estas disciplinas. Las ciencias y disciplinas que integran el plan de estudios deben incorporarse tomando en cuenta los puntos de vinculación entre ellas.

Cada programa de pregrado debe definir con precisión los conocimientos, saberes y aprendizajes que de forma intervinculada deben ofrecer los distintos departamentos académicos y en función de ello estructurar el plan de estudios. El tipo y nivel de conocimientos, aprendizajes y habilidades que cada ciencia y disciplina debe incorporar para su aplicación y desarrollo, debe ser definido y delimitado de manera colegiada y correlacionada por todos los departamentos académicos.

Los planes de estudio de los programas de pregrado deben estructurarse con la participación de todos los departamentos académicos del CU de adscripción, adicional a los de otros CU, ello en atención a un esquema académico matricial y transversal de carácter integral. 
Estas acciones permitirán incorporar al plan de estudios el dominio de habilidades y conocimientos sobre la disciplina eje, así como sus interrelaciones con otras, y por consiguiente, que el egresado adquiera el conjunto de competencias requeridas para el desempeño profesional.

La organización académica de tipo matricial debe trabajar fundamentalmente en la incorporación de conocimientos de orden superior y en el desarrollo de habilidades para el tratamiento de problemas de carácter complejo, sustentados en el trabajo de carácter cooperativo y colaborativo. Este tipo de formación debe desarrollarse tanto para el aprendizaje, como para la docencia e investigación. Lo anterior, tomando en cuenta el tipo de programa de pregrado, si es de carácter multidisciplinar, interdisciplinar y/o transdisciplinar.

Por otra parte, la Red Universitaria muestra un alto grado de desvinculación entre los departamentos académicos y los coordinadores de carrera (de programas de pregrado), cuando en teoría deben ser parte de un núcleo integrador centrado en el plan de estudios y sustentado por un lado en las y los docentes y la enseñanza, y por el otro en las y los estudiantes y el aprendizaje.

A nivel Red el nivel de desvinculación académica se evidencia desde el momento en que solo el 35.7\% de los coordinadores de carrera se vinculan con el jefe de departamento académico para tratar asuntos relativos a los programas que coordinan, y el $64.3 \%$ lo hace con otras cinco instancias diferentes, lo cual muestra un alto grado de desvinculación entre jefes de departamento académico y coordinadores de carrera, así como una directriz académica diversa.

Otro situación de desvinculación académica se manifiesta en el hecho de que los coordinadores de carrera no son miembros integrantes de ningún órgano colegiado, ni a nivel de la Red ni a nivel de CU, y solo el 38.5\% de ellos son invitados a participar como asistentes de forma regular a las sesiones de trabajo de dichos órganos, lo que genera un vacío académico importante desde el momento en el cual los responsables de la conducción y desarrollo de los programas de pregrado no son tomados en cuenta en las decisiones académicas.

La participación de los coordinadores de carrera en los órganos colegiados es relevante para el óptimo desarrollo del programa de pregrado. Al respecto el $86.7 \%$ de los coordinadores opinan que su participación en los órganos colegiados debe ser con voz y voto, y el 13.2\% considera que debería de ser solo con voz.

Para eliminar los elementos restrictores señalados, se debe plantear estrategias e integrar mecanismos que abonen al trabajo académico articulado tanto a nivel Red como de CU; que permitan desarrollar el trabajo entre programas de pregrado, entre coordinadores de carrera, entre departamentos académicos, así como entre coordinadores de carrera y departamentos académicos. Algunos mecanismos que pueden permitir la articulación a nivel Red son la constitución de academias en red para desarrollar el trabajo inter academias; la constitución del comité técnico de coordinadores para el trabajo conjunto de los programas de pregrado, el comité inter académico para el trabajo compartido entre coordinadores de carrera y departamentos; así como la instalación de comités curriculares en red para el trabajo curricular de los programas de pregrado compartidos por varios CU.

Otro aspecto a tomar en cuenta es el relacionado con el abordaje pedagógico a incorporar tanto para el desarrollo académico matricial como para el desarrollo de los enfoques académicos de carácter multi, inter y/o transdisciplinar.

El modelo pedagógico debe sustentarse en la enseñanza y aprendizaje de matriz, contemplar el saber intervinculado e interdependiente y tomar en cuenta la convergencia de inteligencias múltiples.

La metodología de enseñanza y aprendizaje deben tomar en cuenta las pedagogías y didácticas desarrolladas bajo la lógica del pensamiento complejo, pues este permite el desarrollo de conocimientos y habilidades de orden superior, el cual toma en cuenta la interrelación de los saberes e integración de disciplinas, en síntesis, que consienten la articulación matricial del conocimiento.

El modelo pedagógico debe incorporar el trabajo colegiado de docentes y trabajo en equipo de estudiantes. Debe incorporar de forma práctica el aprendizaje y enseñanza articulado de las temáticas que integran las unidades del plan de estudios, así como la enseñanza y aprendizaje basados en la transferencia, 
complementariedad y unificación disciplinar; donde profesionales de diferentes áreas y disciplinas trabajen en conjunto para dar solución a los problemas del entorno.

La enseñanza y aprendizaje deben desarrollarse con fundamento en la integración de las ciencias y disciplinas, en donde los docentes estimulen y faciliten los conocimientos y habilidades asociados; que permita la impartición de conocimientos e intercambio de saberes de docentes de otros CU y universidades; la colaboración de especialistas de diversas áreas; y el desarrollo conjunto con otros profesionales.

El modelo académico matricial requiere de la aplicación de un método pedagógico transversal sustentado en la enseñanza de cruces e intersección de conocimientos. Requiere de la incorporación del método de aprendizaje basado en problemas, donde profesionales de diversas disciplinas participen en la solución del problema objeto de estudio. En la solución de los problemas deben colaboran docentes especialistas de diversas disciplinas, y en las prácticas y ejercicios académicos estudiantes de diversas licenciaturas y profesiones.

El modelo de enseñanza y aprendizaje debe permitir el abordaje de los problemas del entorno incorporando una metodología de carácter matricial y transversal, que atienda la necesidad de un currículo que unifica e interrelaciona diversas disciplinas de estudio, el cual integre los saberes que aportan cada ciencia y disciplina para el conocimiento y solución del problema objeto de estudio.

La formación pedagógica y didáctica de docentes debe evolucionar hacia el modelo de clase transversal. La capacitación pedagógica y didáctica del docente debe realizarse atendiendo la organización del conocimiento que implica el desarrollo del modelo matricial y los enfoques inter, multi y/o transdisciplinar. Se trata de una nueva cultura, que conlleva una nueva manera de abordar el aprendizaje y la enseñanza.

En el ámbito del diseño curricular es recomendable que los planes de estudio del pregrado incorporen la estructura modular, en virtud de que los módulos temáticos permiten la enseñanza y aprendizaje especializados, trabajar de mejor manera la interconexión de conocimientos, incorporar a docentes expertos y expertas en las temáticas tratadas, promover la participación de especialistas del interior y exterior de la propia universidad, y por consiguiente, ampliar y fortalecer el enfoque matricial de los programas de pregrado.

El modelo pedagógico transversal requiere de la formación de redes de aprendizaje, enseñanza e investigación integradas por estudiantes, docentes e investigadores de diversas profesiones para el tratamiento integral de los problemas. Asimismo, en materia de investigación la universidad debe constituir centros de investigación para el desarrollo científico y académico de forma clasificada, atendiendo los fines y niveles de complejidad que exponen los enfoques multi, inter y/o transdisciplinar.

El desarrollo del modelo académico matricial requiere incorporar esquemas para la vinculación interna y externa; establecer esquemas de vinculación académica y profesional con otras universidades, instituciones de educación superior, centros de investigación y organizaciones públicas y privadas del país y del extranjero; constituir redes y comunidades de aprendizaje entre estudiantes, de enseñanza entre docentes, de investigación entre investigadores y combinadas entre todas.

En la Universidad de Guadalajara (en su Red Universitaria) los problemas que restringen la matricialidad académica e impiden la plena incorporación de los enfoques multi, inter y/o transdisciplinar muestran a su vez áreas de oportunidad para la mejora de dichos aspectos. Entre estos se encuentran los relacionados con la legislación universitaria, gestión académica y administración educativa.

Con respecto a la legislación universitaria, el $50.5 \%$ de los coordinadores de carrera manifiesta realizar funciones adicionales a las especificadas, y el 84.5\% coincide en que la legislación debe actualizarse. Los principales aspectos a incorporar en la normatividad están relacionados con la redefinición de sus funciones y atribuciones, así como lo relativo a su inclusión en los órganos colegiados, principalmente en el colegio departamental eje y academias.

En el ámbito de la gestión académica los coordinadores de carrera consideraron que esta no permite incorporar plenamente el programa de pregrado al modelo departamental de carácter matricial. Al preguntar lo que se requiere para lograr este cometido, las respuestas fueron las siguientes: Gestión más efectiva por 
parte de autoridades y jefes de departamento académico (8.69\%); mayor apoyo de departamentos académicos y autoridades hacia las coordinaciones de carrera (19.5\%); mayor articulación funcional entre coordinación de carrera y departamentos académicos (32.6\%); que la oferta de unidades de aprendizaje sea más incluyente con los departamentos académicos del CU (34.7\%); otras acciones (4.34\%).

En el ámbito de la gestión los coordinadores de programas de pregrado deben trabajar en dos vertientes, una hacia el interior de los CU y otra a nivel de toda la Red Universitaria. Al interior de los CU deben elaborar un plan de gestión que: a) Ubique las actividades a desarrollar de forma planeada y ordenada, b) Indique el tiempo en el que se van a realizar las actividades, con temporalidades y plazos definidos, c) Identifique los actores con los que deben realizarse las acciones y diligencias de gestión, d) Identifique los mecanismos e instrumentos a su alcance para el logro de los objetivos y metas proyectados, y f) Valore la contribución, logros e impacto de la gestión en los programas de pregrado. A nivel de la Red Universitaria se debe constituir el comité técnico de coordinadores programas de pregrado que tenga entre otras, las funciones siguientes: a) Atender asuntos académicos comunes a los programas de pregrado de la Red Universitaria, b) Orientar el trabajo académico a nivel Red inherente a los programas de pregrado, c) Proponer acciones que permitan atender las asimetrías que presentan los programas de pregrado de la Red, d) Sugerir acciones para fortalecer las capacidades de los coordinadores de pregrado de la Red, e) Plantear estrategias que permitan el incremento de los indicadores de desempeño relacionados con los programas de pregrado de la Red, f) Establecer pautas para fortalecer la articulación académica entre las coordinaciones de pregrado y los departamentos académicos a nivel Red, y g) Incorporar elementos constitutivos de calidad a los programas de pregrado.

En relación con la administrativa educativa, el 56.5\% de coordinadores de carrera manifestó no contar con apoyo de personal para el desarrollo de sus funciones, y el $47.5 \%$ señalo que su CU de adscripción no cuenta con un coordinador general de coordinadores de programas de pregrado. El diseño de una estructura de personal para las coordinaciones de carrera en función de la matrícula de cada programa de pregrado, la asignación de un coordinador general de coordinadores por CU y la elaboración del manual de organización y procedimientos de las coordinaciones de programas de pregrado, redundaría en óptimos beneficios para el desarrollo académico.

El modelo departamental de carácter matricial juega un papel preponderante para la incorporación y desarrollo de los enfoques multi, inter y/o transdisciplinar, por ello la Universidad de Guadalajara (su red Universitaria) debe plantearse el establecimiento de mejores condiciones para la consolidación de dicho modelo. El reto radica en implementar acciones orientadas a la actualización normativa, organizacional, curricular, formativa, pedagógica y de gestión.

En síntesis, la Red Universitaria de Jalisco necesita implementar un conjunto de estrategias, mecanismos y acciones que le permitan consolidar el modelo académico en Red, corregir el avance asimétrico del modelo departamental y fortalecer el funcionamiento académico de carácter matricial con el fin de establecer las condiciones que accedan a incorporar plenamente los enfoques formativos de carácter multidisciplinar, interdisciplinar y/o transdisciplinar.

\section{REFERENCIAS}

ANUIES. (2018). Visión y acción 2030. Propuesta de la ANUIES para renovar la educación superior en México. Diseño y concertación de politicas públicas para impulsar el cambio institucional 31 y 42. México: ANUIES Recuperado de https://visionyaccion2030.anuies.mx/Vision_accion2030.pdf

Ardines, P. (s.f.). El trabajo multidisciplinario, nuevo enfoque para la solución de problemas y para la realización de programas en el área de la salud. Recuperado de: http://www.posgrado.unam.mx/publicaciones/ ant_omnia/11/14.pdf 
Bravo, I. T. (2017). Estadística Institucional 2016-2017. Jalisco, México: Universidad de Guadalajara. Recuperado de https://goo.gl/uYXFFx

Congreso de los Estados Unidos Mexicanos. (1978). Ley para la Coordinación de la Educación Superior, 1. México. Recuperado de https://goo.gl/KFbnBm

Del Rio, F. (2002). El funcionamiento matricial o departamental en las Instituciones de Educación Superior. Las DES como instrumentos de planeación de las IES en México, 1-2. Recuperado de https://goo.gl/fFVt1V

Escobar, Y. (2010). Interdisciplinariedad desafío para la Educación Superior y la Investigación. Revista Luna Azul de la Universidad de Caldas, 31, 156-169. Recuperado de http://www.scielo.org.co/pdf/luaz/n31/n31a11.pdf

Luengo, E. (2003). Tendencias de la Educación Superior en México: Una lectura desde la perspectiva de la complejidad. Bogotá, Colombia. Recuperado de https://goo.gl/aa7N41

Luengo, E. (Coord.). (2012). Interdisciplina y transdisciplina: aportes desde la investigación y la intervención social universitaria. La transdisciplina y sus desafíos a la universidad. Guadalajara, Jalisco, México: ITESO. Recuperado de https://goo.gl/4dESdJ

Thompson, J., Grossenbacher-Mansuy, W., Haberli, R., Bill, A., Scholz, R. W. y Welti, M. (eds.). (2001). Transdisciplinarity: joint problem solving among science, technology and society. Basilea: Birkhauser.

UNESCO. (2009). Conferencia mundial sobre la Educación Superior - 2009: La nueva dinámica de la educación superior y la investigación para el cambio social y el desarrollo. París, Fracia UNESCO. Recuperado de https://goo.gl/ P9oS4W

Universidad de Guadalajara. (1994). Ley Orgánica de la Universidad de Guadalajara. Recuperado de https://goo.gl/ iGYY8K

\section{Notas}

[1] La Universidad de Guadalajara registró para el periodo 2016-2017 una matrícula de 270.309 estudiantes, de los cuales 150.011 pertenecen al nivel medio superior (bachillerato); 113.992 al nivel superior (pregrado) y 6.306 al posgrado (Bravo, 2017, p. 24).

[2] El término matricialidad se usa para hacer referencia al número de departamentos académicos de un centro universitario que integran y ofrecen asignaturas al plan de estudios de un programa de pregrado.

[3] La Ley Orgánica de la Universidad de Guadalajara (LOUDG) en su artículo 23 señala en la fracción I, que la Red Universitaria se integrará por Centros Universitarios que podrán ser temáticos o regionales, y establece las definiciones siguientes: a) Centros Temáticos, los que organicen y administren sus programas académicos, con base en áreas afines del conocimiento o en campos del ejercicio profesional, y b) Centros Regionales, los que organicen y administren sus programas académicos, en atención a necesidades regionales multidisciplinarias. También señala en la fracción II, que los Centros Universitarios estarán integrados por Divisiones y Departamentos, y establece las definiciones siguientes: a) Las Divisiones, serán las entidades académico-administrativas que agruparán un conjunto de Departamentos; b) Los Departamentos, serán las unidades académicas básicas, en donde se organicen y administren las funciones universitarias de docencia, investigación y difusión (Universidad de Guadalajara, 1994, pp.6-7).

[4] Se utilizó el denominado benchmarking interno, en virtud de que este permite comparar actividades similares en diferentes áreas, departamentos o unidades operativas de una misma organización.

[5] Se aplicó la encuesta a los coordinadores de carrera (de programas de pregrado) por ser los funcionarios responsables de hacer realidad la concreción del currículum, porque el papel que desempeñan se relaciona directamente con todo el proceso formativo de las y los estudiantes, desde el ingreso, hasta el egreso y titulación; y por consiguiente, por considerar que tienen una visión integral del programa académico que coordinan.

[6] Se consideró un coordinador por cada programa de pregrado, con la finalidad de obtener una encuesta por cada programa, ello independientemente de los coordinadores que tienen a su cargo más de un programa educativo.

[7] Un programa de pregrado es de carácter exclusivo cuando lo oferta e imparte un solo CU, y se considera compartido cuando se ofrece e imparte por más de un CU de la Red Universitaria.

\section{BY-NC-ND}

